\section{Efeito da duração da amamentação exclusiva e mista sobre os níveis de hemoglobina nos primeiros seis meses de vida: um estudo de seguimento}

\author{
Effect of length of exclusive breastfeeding and \\ mixed feeding on hemoglobin levels in the \\ first six months of life: a follow-up study
}

\author{
1 Universidade Federal da \\ Bahia, Salvador, Brasil. \\ 2 Secretaria de Saúde do \\ Estado da Bahia, Salvador, \\ Brasil. \\ Correspondência \\ A. S. Oliveira \\ Universidade Federal da \\ Bahia. \\ Conjunto Colinas de \\ Pituaçu, bloco 379, apto. 104 \\ Salvador, $B A$ \\ 41250-520, Brasil. \\ dedanut1@yahoo.com.br
}

\begin{abstract}
This six-month follow-up study aimed to assessing the effect of length of both exclusive breastfeeding and mixed feeding on hemoglobin levels in 150 infants born at the Mutuipe maternity facility in Bahia State, Brazil. Blood tests were performed monthly to assess the hemoglobin levels, at which time data were gathered on food intake. Obstetric and socioeconomic data were also col lected. The linear mixed effects model was used to investigate the target associations. Loss to follow up was $15.3 \%$, and anemia prevalence was $71.7 \%$. An increase of $0.091 \mathrm{~g} / \mathrm{dL}(p=0.031)$ in hemoglobin levels was found for each month of exclusive breastfeeding, while a decrease of $0.097 \mathrm{~g} / \mathrm{dL}$ ( $p=$ 0.017) was shown for each month of mixed feeding. In conclusion, inadequate food practices appear to be directly involved in the etiology of iron deficiency anemia during infancy.
\end{abstract}

Breast Feeding; Infant Nutrition; Anemia
Andréa Silva de Oliveira 1,2 Rita de Cássia Ribeiro Silva 1 Rosemary Leovigildo Fiaccone 1 Elizabete de Jessus Pinto 1 Ana Marlúcia Oliveira Assis 1

\section{Introdução}

A anemia nutricional caracteriza-se, segundo a Organização Mundial da Saúde (OMS), pela concentração, no sangue, de hemoglobina abaixo dos valores de referência para idade e sexo, em conseqüência da carência de um ou mais nutrientes essenciais ao organismo humano, tais como o ferro, o ácido fólico, a vitamina B12 e a vitamina A 1. A deficiência de ferro é responsável por $90 \%$ dos dois bilhões de casos de anemia estimados em todo o mundo, constituindo-se, hoje, na mais freqüente das deficiências nutricionais 2 .

As gestantes e as crianças compõem os grupos de maior vulnerabilidade à carência de ferro. Nos países desenvolvidos, $22,7 \%$ das gestantes e $20,1 \%$ das crianças pré-escolares são afetadas, enquanto nos países em desenvolvimento esses percentuais se elevam para $52 \%$ e $39 \%$, respectivamente ${ }^{3}$. Em algumas regiões da América do Sul e na América Central, a anemia ferropriva chega a atingir mais de $50 \%$ das gestantes e das crianças 4 .

No Brasil, os estudos pontuais revelam o caráter endêmico dessa deficiência. Entre os préescolares, foram registradas prevalências de $36,4 \%$ a $96,4 \%$, em diversas regiões $5,6,7,8,9,10$. Já entre os lactentes, as prevalências de anemia ferropriva têm sido estimadas entre $50 \%$ e $73,2 \%$ $11,12,13,14,15$.

A anemia por deficiência de ferro constitui um importante problema de saúde pela eleva- 
da prevalência e efeitos deletérios que impõe ao organismo. Na infância, tem sido identificado o efeito negativo sobre a imunidade celular 16, o crescimento, o desenvolvimento psicomotor 17 , a função cognitiva e a aprendizagem 18. Essas conseqüências, que variam de acordo com a gravidade e a duração da anemia, podem persistir mesmo após a correção da deficiência 19 .

$\mathrm{O}$ armazenamento de ferro realizado durante a vida intra-uterina garante o atendimento das necessidades do lactente nos seis primeiros meses de vida ${ }^{20}$. Nesse período, é sugerido que o leite materno oferecido de forma exclusiva seja suficiente para manter um estado nutricional adequado em relação ao ferro 21 . No entanto, vários fatores estão envolvidos na etiologia da anemia ferropriva entre os lactentes, em especial as baixas reservas de ferro ao nascer, principalmente quando esse fato se associa ao oferecimento precoce de alimentos com inadequado teor desse mineral 22 .

Entre as crianças brasileiras, tem sido observado padrão alimentar caracterizado pela substituição precoce do leite humano pelo leite de vaca e, ainda, pela adição de outros alimentos com baixa biodisponibilidade de ferro $23,24,25$.

A quantidade e a qualidade dos alimentos que compõem a dieta da criança associam-se com os níveis de hemoglobina. Resultados de algumas investigações indicam que o aleitamento materno exclusivo, nos primeiros seis meses de vida, assegura níveis de hemoglobina mais elevados, quando comparado aos demais regimes alimentares, e que os níveis de hemoglobina se tornam compatíveis com a anemia quando o leite de vaca passa a integrar o regime alimentar da criança 14,25. Outros estudos destacam uma possível relação entre o consumo de leite de vaca e a anemia em crianças, ao associar o aumento da participação desse tipo de leite nas calorias totais da dieta com o aumento da prevalência de anemia e a diminuição da concentração da hemoglobina 26,27,28.

Contudo, pesquisas que avaliem as mudanças dos níveis de hemoglobina no curso do crescimento, levando em consideração o tipo do regime alimentar adotado, são escassas. Assim, com a presente investigação, espera-se contribuir para o conhecimento sobre o efeito dos tipos de regimes alimentares sobre os níveis de hemoglobina durante os seis primeiros meses de vida da criança.

\section{Métodos e técnicas \\ Desenho e população do estudo}

Este estudo é parte de uma investigação mais ampla intitulada Amamentação e Alimentação Complementar no Desmame - Estado de Nutrição e Saúde nos Dois Primeiros Anos de VidaUm Estudo de Coorte, desenvolvida pela Escola de Nutrição da Universidade Federal da Bahia (ENUFBA).

Trata-se de um estudo envolvendo 150 crianças acompanhadas nos primeiros seis meses de vida, no período de junho de 2005 a outubro de 2006, na cidade de Mutuípe, Bahia.

O município, localizado a 235km de Salvador, na região do Recôncavo Sul do Estado da Bahia, conta com 20.462 habitantes, sendo 11.478 (56\%) domiciliados na área rural e 8.984 (44\%), na área urbana. Predominam, no município, as atividades agrárias, destacando-se o cultivo de cacau, banana, mandioca, feijão e milho ${ }^{29}$. No plano econômico, o município encontra-se na 146a posição dentre os 417 municípios do estado, de acordo com o Índice de Desenvolvimento Econômico (IDE) da Superintendência de Estudos Econômicos (SEI/BA) 30 .

Considerando que o tamanho da amostra não foi calculado para avaliar a associação entre os níveis de hemoglobina e os tipos de regimes alimentares, procedeu-se ao cálculo do poder da amostra para avaliar tal relação. Tomou-se como referência a diferença de $10 \%$ entre o primeiro e o sexto mês de vida. O resultado da análise indicou que a amostra de 150 crianças, considerando-se $15,3 \%$ de perda, tinha poder $(Z=4,627$; poder $=99 \%)$ para avaliar a diferença nos níveis de hemoglobina, conforme descrito anteriormente.

\section{Critérios de elegibilidade}

Compuseram a população deste estudo as crianças nascidas na maternidade da cidade, residentes nas áreas urbana e rural do município, que não apresentavam defeitos de conformação da cavidade oral.

\section{Coleta de dados}

A coleta dos dados durou 16 meses (10 de captação e 6 de seguimento) e foi realizada por nutricionistas treinados. A primeira consulta foi efetuada no domicílio da criança no final do primeiro mês, e as demais aconteceram mensalmente no posto de saúde do município, conforme agendamento prévio. 


\section{Variáveis estudadas}

A determinação da concentração de hemoglobina foi realizada utilizando-se o hemoglobinômetro portátil Hemocue, por meio do método de cianeto hemoglobina 3 . Para efetuar a dosagem da hemoglobina ao nascer, utilizou-se o sangue do cordão, e as dosagens mensais foram realizadas por punctura digital. Em caso de aferição da hemoglobina com valor menor que $9 \mathrm{~g} / \mathrm{dL}$, efetuou-se a segunda dosagem, e a média entre as duas medições foi adotada como medida definitiva ${ }^{31}$. As medidas dos níveis de hemoglobina constituem a variável dependente do modelo em estudo.

O consumo alimentar foi investigado mensalmente, aplicando-se o questionário de registro alimentar. Para que este fosse caracterizado, foi utilizada a definição proposta pela Organização Pan-Americana da Saúde (OPAS) 32, considerando cinco tipos de regimes alimentares:

- amamentação materna exclusiva (AME): regime alimentar integrado somente por leite materno;

- amamentação materna predominante (AMP): leite materno como única fonte láctea, associado a outros líquidos, como água, chá ou sucos; - amamentação materna complementada (AMC): leite materno como única fonte láctea, complementado por outros alimentos;

- amamentação mista (AM): leite materno e leite de vaca, independentemente do consumo ou não de outros alimentos;

- amamentação artificial (AA): leite de vaca como única fonte láctea, podendo ou não incluir outros alimentos.

O regime alimentar, especificado em cinco tipos, foi adotado como variável independente principal, e a duração de cada tipo foi calculada com base no tempo (em meses) em que cada criança permaneceu no regime.

A idade da criança foi obtida em dias, pelo cálculo da diferença entre a data da entrevista e a data do nascimento. Para efeito de análise, a idade foi convertida em meses.

A medida do peso ao nascer foi obtida utilizando-se a balança pediátrica microeletrônica, marca Fillizola (São Paulo, Brasil), modelo E150/3P, com precisão de $10 \mathrm{~g}$. A aferição foi realizada em duplicata 33 , procedendo-se segundo as técnicas descritas por Lohman et al. 34 .

As informações sociais, econômicas e ambientais são representadas por idade e escolaridade (anos de escolaridade) maternas; zona de residência (rural e urbana); saneamento ambiental do domicílio (abastecimento de água, esgoto e coleta pública de lixo) e condições de moradia (tipo de construção, número de pessoas residen- tes, número de dormitórios e presença de instalação sanitária).

Essas variáveis deram origem a um índice denominado "condições de vida", adaptado do modelo proposto por Issler \& Giugliani 35. A cada situação foi atribuída uma pontuação; a mais favorável recebeu o valor 0 e a mais desfavorável, a pontuação 4, resguardando os valores intermediários. O somatório desses valores caracterizou as condições de vida.

Na modelagem estatística, todas as variáveis foram empregadas na forma contínua.

\section{Perdas de seguimento}

As perdas amostrais são comuns em estudos de seguimento. O teste $\chi^{2}$ foi utilizado para comparar as proporções entre os grupos perdidos e os seguidos em cada fase do acompanhamento em relação às variáveis ligadas diretamente a crianças, como sexo (masculino, feminino); peso ao nascer (baixo: $<2.500 \mathrm{~g}$, insuficiente: 2.500-2.999g, normal: $\geq 3.000 \mathrm{~g}$ ); idade gestacional (pré-termo: $<37$ semanas, a termo: $\geq 37$ semanas) e tipo de parto (normal e cesáreo). Quando as categorias tiveram um $\mathrm{n}$ menor do que 5 , foi aplicado o teste exato de Fischer.

\section{Modelagem estatística}

O modelo linear de efeitos mistos (MLEM) foi adotado neste estudo para avaliar a relação de interesse por considerar a variabilidade do nível de hemoglobina entre e intra-indivíduos. Esse modelo permite levar em conta os dados desbalanceados, considera o intercepto e/ou os coeficientes do modelo de regressão como efeitos aleatórios e incorpora a estrutura de dependência referente às observações acerca de um mesmo indivíduo, controlando essa variação e produzindo estimativas mais precisas 36 . Dessa forma, foram construídos cinco modelos de regressão, um para cada variável independente (regime alimentar), considerando-se, inicialmente, para cada modelo, o ajuste de um modelo reduzido. Em seguida, examinou-se a importância da introdução de cada uma das variáveis explicativas e sua interação com as demais variáveis do modelo. $\mathrm{O}$ resultado menor do que $25 \%$, critério sugerido por Hosmer \& Lemeshow 37 , foi considerado como um provável termo de interação.

No modelo final, foram aceitas como estatisticamente significantes as associações que apresentaram valor de $\mathrm{p}<0,05$. Utilizou-se o critério Akaike Information Criterion (AIC) para a escolha da melhor estrutura a ser adotada no modelo em questão. $O$ teste da razão de verossimilhança 
também foi empregado na comparação de modelos ajustados.

Em virtude de os dados em questão apresentarem uma estrutura desbalanceada pela presença de dados omissos, algumas ferramentas gráficas, como o gráfico das médias suavizadas e o variograma (não apresentados), foram utilizados para avaliar a melhor estrutura de correlação a ser adotada no modelo de lineares de efeitos mistos. Assim, a matriz de correlação auto-regressiva (AR1) foi a que melhor se adequou aos dados.

Os dados foram analisados utilizando-se o pacote estatístico Stata, versão 9.0 (Stata Corp., College Station, Estados Unidos).

\section{Aspectos éticos}

A pertinência ética do estudo foi atestada pelo Comitê de Ética do Hospital Universitário Professor Edgard Santos (HUPES), da Universidade Federal da Bahia (UFBA). As gestantes que concordaram em participar do estudo assinaram o termo de consentimento livre e esclarecido e foram orientadas para a adoção da alimentação infantil de acordo com as recomendações do Ministério da Saúde ${ }^{38}$. Durante o seguimento, as crianças diagnosticadas com problemas de saúde foram encaminhadas ao serviço de saúde.

\section{Resultados}

A população em estudo constituiu-se de 150 crianças, que foram acompanhadas nos seis primeiros meses de vida. A distribuição da população segundo sexo indicou uma leve predominância de meninas na amostra $(50,7 \%)$. O baixo peso ao nascer foi observado em $5,3 \%$ das crianças, e $26,7 \%$ nasceram com peso considerado insuficiente. A idade gestacional média foi de 38,54 semanas, variando de 33 a 42 semanas. A maioria das crianças nasceu a termo (94\%) e de parto normal (82\%); $25 \%$ residiam em melhores condições (Tabela 1).

Das 150 crianças que iniciaram o acompanhamento, 127 completaram as seis entrevistas previstas, perfazendo uma perda definitiva de $15,3 \%$. As características relacionadas às crianças perdidas e acompanhadas mostraram-se similares, indicando o caráter aleatório das perdas de seguimento.

Na amostra estudada, a média da hemoglobina ao nascer foi de 15,34g/dL, variando de 9,5g/ dL a 22g/dL. Observou-se, até o segundo mês, um declínio acentuado nas concentrações de hemoglobina, chegando ao valor médio de 10,18g/ $\mathrm{dL}( \pm 1,18 \mathrm{~g} / \mathrm{dL})$. A partir daí, permaneceram praticamente constantes, atingindo $10,3 \mathrm{~g} / \mathrm{dL}$
$( \pm 1,16 \mathrm{~g} / \mathrm{dL})$ ao fim dos seis meses de vida. No sexto mês, os valores médios de hemoglobina foram menores do que o adotado pela OMS $(<11 \mathrm{~g} /$ dL) 39 como referência de normalidade, verificando-se, nesse período, $71,7 \%$ de anemia entre as crianças estudadas.

No primeiro mês de vida, 97,3\% das crianças foram aleitadas ao seio materno, e 56,8\% tinham introduzido líquidos como chás e água na alimentação. Nesse período, foi iniciado o uso de leite de vaca integral por $24,3 \%$; de farináceos, por $16,9 \%$; de açúcar por $34,5 \%$ e de frutas, por $4,15 \%$ das crianças estudadas. No segundo mês, as verduras foram introduzidas na alimentação de $11,2 \%$ delas. A partir de então, notou-se o aumento crescente do consumo de leite de vaca integral, açúcar, farinhas e massas, frutas e verduras, e a redução do uso do leite materno na alimentação das crianças. No quarto mês, 1,3\% das crianças iniciou o consumo de carnes. No sexto mês, o percentual de crianças que consumiam leguminosas e carnes era de $12 \%$ e $15,3 \%$, respectivamente.

Na Tabela 2, apresentam-se os resultados dos modelos estatísticos para a avaliação do comportamento dos níveis de hemoglobina segundo o regime alimentar. Após o ajuste por idade, peso ao nascer e condições de vida, observou-se que, quanto maior a duração do aleitamento materno exclusivo, maiores foram os níveis de hemoglobina, com incremento mensal de 0,099g/dL $(p=0,027)$. Coeficiente negativo e significante foi detectado para o regime de aleitamento misto, com o decréscimo de $0,095 \mathrm{~g} / \mathrm{dL}(\mathrm{p}=0,025)$ nos níveis de hemoglobina, a cada mês de duração desse regime alimentar.

\section{Discussão}

Os resultados da presente investigação apontam para o efeito benéfico do aleitamento materno exclusivo nos seis primeiros meses de vida, por conferir o incremento de $0,099 \mathrm{~g} / \mathrm{dL}$ por mês nos níveis de hemoglobina das crianças expostas. Identificou-se, também, que a associação do aleitamento materno com o uso do leite de vaca provocou o declínio dos níveis de hemoglobina das crianças no mesmo período observado ( $\beta=$ $-0,095 \mathrm{~g} / \mathrm{dL} ; \mathrm{p}=0,025)$. Tais resultados corroboram os de outros estudos, que constataram concentrações mais elevadas de hemoglobina entre as crianças em aleitamento materno exclusivo 14,40,41 e a diminuição dos níveis de hemoglobina com o aumento da participação do leite de vaca na dieta das crianças 26,27,28.

Dessa forma, especula-se que o benefício conferido pelo leite materno sobre os níveis de 
Características demográficas e sócio-ambientais de crianças menores de seis meses. Mutuípe, Bahia, Brasil, 2007.

\begin{tabular}{|c|c|c|}
\hline Variáveis & $\mathrm{n}$ & $\%$ \\
\hline \multicolumn{3}{|l|}{ Sexo da criança } \\
\hline Masculino & 74 & 49,3 \\
\hline Feminino & 76 & 50,7 \\
\hline \multicolumn{3}{|l|}{ Peso ao nascer (g) } \\
\hline Baixo: $<2.500$ & 8 & 5,3 \\
\hline Insuficiente: $2.500-2.999$ & 40 & 26,7 \\
\hline Normal: $\geq 3.000$ & 102 & 68,0 \\
\hline \multicolumn{3}{|l|}{ Idade gestacional (semanas) * } \\
\hline Pré-termo $<37$ & 7 & 4,7 \\
\hline A termo $\geq 37$ & 141 & 94,0 \\
\hline \multicolumn{3}{|l|}{ Tipo de parto (b) } \\
\hline Normal & 123 & 82,0 \\
\hline Cesáreo & 26 & 17,3 \\
\hline \multicolumn{3}{|l|}{ Idade materna (anos) ** } \\
\hline$<20$ & 53 & 35,3 \\
\hline $20-34$ & 90 & 60,0 \\
\hline$\geq 35$ & 6 & 4,0 \\
\hline \multicolumn{3}{|l|}{ Escolaridade materna } \\
\hline Fundamental & 119 & 79,3 \\
\hline Médio ou + & 32 & 20,7 \\
\hline \multicolumn{3}{|l|}{ Zona de residência } \\
\hline Urbana & 55 & 36,7 \\
\hline Rural & 95 & 63,3 \\
\hline \multicolumn{3}{|c|}{ Índice de condições de moradia } \\
\hline Elevado & 38 & 25,3 \\
\hline Intermediário & 61 & 40,7 \\
\hline Baixo & 22 & 14,7 \\
\hline Muito baixo & 29 & 19,3 \\
\hline
\end{tabular}

* Duas perdas de seguimento;

** Uma perda de seguimento.

hemoglobina é anulado pelo leite de vaca quando este é oferecido à criança durante o período de amamentação ao seio. Sabe-se que o leite de vaca integral exerce influência negativa na concentração de hemoglobina pela baixa concentração de ferro e alto teor de cálcio e caseína, fatores inibidores da absorção do mineral 42,43 . O leite de vaca pode ainda ocasionar sangramento oculto nas fezes, principalmente nos primeiros meses de vida, integrando, assim, a lista de alimentos de efeito negativo sobre o estado nutricional de ferro nos lactentes 42 .

$\mathrm{O}$ aleitamento materno adotado exclusivamente nos seis primeiros meses de vida é reconhecido como padrão alimentar capaz de garantir a saúde da criança nesse período, em virtude da composição nutricional e dos fatores de proteção contra doenças que esse alimento confere 42 . No leite materno, o ferro encontra-se em baixa concentração, porém com uma biodisponibilidade elevada (cerca de 50\%), o que garante a utilização suficiente para atender à demanda do mineral nos primeiros seis meses de vida $38,39,44$.

Os regimes de aleitamento materno predominante $(\beta=0,32 ; \mathrm{p}=0,53)$, aleitamento complementado ( $\beta=0,008 ; \mathrm{p}=0,93$ ) e aleitamento exclusivo à base de leite de vaca $(\beta=-0,001 ; p=$ $0,985)$ não se associaram estatisticamente com os níveis de hemoglobina (modelos não apresentados). Contudo, não é possível ignorar os riscos da introdução precoce de alimentos na população em estudo. As evidências indicam que, na maioria das vezes, os alimentos complementares - em especial aqueles à base de frutas - são altamente diluídos e de baixa densidade de energia e micronutrientes, sendo insuficientes para atender às demandas nutricionais da criança e, em particular, os requerimentos de ferro $23,24,45$. 
Tabela 2

Modelo linear de efeitos mistos para os níveis de hemoglobina ( $\mathrm{g} / \mathrm{dL}$ ) de crianças menores de seis meses, segundo amamentação exclusiva e mista. Mutuípe, Bahia, Brasil, 2007.

\begin{tabular}{|c|c|c|c|}
\hline \multicolumn{4}{|c|}{ ALEITAMENTO MATERNO EXCLUSIVO } \\
\hline Variável & Estimativa & Erro padrão & Valor de p \\
\hline \multicolumn{4}{|l|}{ Efeitos fixos } \\
\hline Intercepto & 14,177 & 0,573 & 0,000 \\
\hline Idade (meses) & $-2,569$ & 0,095 & 0,000 \\
\hline Efeito quadrático da idade (meses) & 0,316 & 0,015 & 0,000 \\
\hline Duração da amamentação exclusiva (meses) & 0,099 & 0,044 & 0,027 \\
\hline \multirow[t]{2}{*}{ Variável } & & $\mathrm{IC} 95 \%$ & \\
\hline & Limite inferior & Estimativa & Limite superior \\
\hline \multicolumn{4}{|l|}{ Efeitos aleatórios } \\
\hline$\sigma$ intercepto & 0,360 & 0,569 & 0,726 \\
\hline$\sigma$ residual & 1,576 & 1,491 & 1,568 \\
\hline -2 Log-verossimilhança & & $-1749,49$ & \\
\hline $\mathrm{AIC}$ & & 3516,98 & \\
\hline \multicolumn{4}{|c|}{ ALEITAMENTO MATERNO MISTO } \\
\hline Variável & Estimativa & Erro padrão & Valor de p \\
\hline \multicolumn{4}{|l|}{ Efeitos fixos } \\
\hline Intercepto & 14,351 & 0,582 & 0,000 \\
\hline Idade (meses) & $-2,567$ & 0,095 & 0,000 \\
\hline Efeito quadrático da idade (meses) & 0,317 & 0,015 & 0,000 \\
\hline Duração da amamentação mista (meses) & $-0,095$ & 0,042 & 0,025 \\
\hline \multirow[t]{2}{*}{ Variável } & & $1 \mathrm{C} 95 \%$ & \\
\hline & Limite inferior & Estimativa & Limite superior \\
\hline \multicolumn{4}{|l|}{ Efeitos aleatórios } \\
\hline$\sigma$ intercepto & 0,354 & 0,267 & 0,525 \\
\hline$\sigma$ residual & 1,575 & 1,478 & 1,644 \\
\hline -2 Log-verossimilhança & & $-1749,34$ & \\
\hline $\mathrm{AIC}$ & & 3517,01 & \\
\hline
\end{tabular}

IC95\%: intervalo de 95\% de confiança.

AIC: Akaike Information Criterion.

Nas crianças pesquisadas, os valores médios de hemoglobina foram de $15,34 \mathrm{~g} / \mathrm{dL}$ ao nascer, declinando para 10,19g/dL no segundo mês, com pequena variação do terceiro até o sexto mês. A queda acentuada dos níveis de hemoglobina, observada nos dois primeiros meses de vida, pode ser creditada também ao evento de adaptação extra-uterina, denominado anemia fisiológica do lactente ${ }^{21}$. Alguns autores sugerem que, em crianças alimentadas exclusivamente com leite materno, a eritropoiese se torna mais ativa a partir da oitava semana, elevando os valores hematológicos do lactente 21,43 . Portanto, a interrupção precoce do aleitamento materno exclusivo pode resultar em maior vulnerabilidade da criança ao desenvolvimento da deficiência de ferro nos primeiros meses de vida 14,25.

Neste estudo, observou-se a redução progressiva do consumo do leite materno e o consumo precoce de alimentos pobres em ferro. A freqüência do aleitamento materno exclusivo, ao longo do tempo, teve comportamento semelhante ao identificado em estudo multicêntrico realizado pelo Ministério da Saúde, no qual se observou brusca redução em sua prevalência ${ }^{44}$. A duração mediana do aleitamento materno exclusivo, 
detectada na presente investigação, foi de 41,4 dias, muito distante dos 180 dias preconizados pela OMS e pelo Ministério da Saúde, resultado semelhante ao identificado em outras pesquisas $23,46,47,48,49$.

Ao final do seguimento, a anemia ferropriva constituiu um importante problema de saúde e nutrição para os lactentes investigados. Considerando o ponto de corte $(\mathrm{Hb}<11 \mathrm{~g} / \mathrm{dL})$ estabelecido pela OMS, identificou-se a prevalência de $71,7 \%$ de anemia no sexto mês de vida. Em outras pesquisas, para a mesma faixa etária, as prevalências variaram de $28,43 \%$ a $58 \% 8,14,25,50$.

Investigações longitudinais comumente apresentam algumas limitações. No presente estudo, a ocorrência das perdas de seguimento poderia implicar problemas de viés na amostra investigada. Todavia, constatou-se que as perdas foram aleatórias, segundo os resultados das análises processadas. Do mesmo modo, o fornecimento das orientações sobre alimentação adequada poderia influenciar na obtenção dos dados de consumo alimentar das crianças e gerar o viés de informação. No entanto, a omissão desse atendimento feriria os preceitos da ética em um estudo de acompanhamento.

Conclui-se que a anemia ferropriva se apresentou como um grave problema de saúde e nutrição para as crianças acompanhadas e que elas se encontravam em situação de alto risco para a doença nos primeiros meses de vida. As práticas alimentares inadequadas parecem estar diretamente envolvidas na etiologia da deficiência nutricional de ferro nesse período.

Este estudo reforça a necessidade de uma alimentação saudável na infância. Devem-se estimular o desenvolvimento e a manutenção de ações de promoção, proteção e apoio ao aleitamento materno em todos os níveis de atenção, visando à manutenção da amamentação exclusiva nos seis primeiros meses de vida e à adequada introdução dos alimentos complementares no tempo oportuno. A adoção dessa prática é importante para a manutenção da saúde infantil, especialmente pelo efeito protetor que o aleitamento materno exclusivo confere na prevenção da anemia ferropriva durante o primeiro semestre de vida.

\section{Resumo}

O objetivo deste estudo foi avaliar o efeito da duração da amamentação exclusiva e mista sobre os níveis de hemoglobina de lactentes. Trata-se de um estudo de seguimento com seis meses de duração, envolvendo 150 crianças nascidas na maternidade de Mutuípe, Bahia, Brasil. Mensalmente, as crianças eram submetidas ao exame de sangue para avaliação dos níveis de hemoglobina e realizava-se coleta de dados de consumo alimentar. Foram obtidos, ainda, dados obstétricos e sócio-econômicos. Utilizou-se o modelo linear de efeitos mistos para investigar as associações de interesse. Ao final do seguimento, a perda amostral foi de 15,3\%, e detectou-se uma prevalência de 71,7\% de anemia. Constatou-se um incremento de 0,091g/dL nos níveis de hemoglobina a cada mês de duração de aleitamento materno exclusivo ( $p=0,031)$, enquanto, a cada mês de duração de aleitamento materno misto, os níveis de hemoglobina declinaram em 0,097g/dL ( $p=0,017$. Concluiu-se que as práticas alimentares inadequadas parecem estar diretamente envolvidas na etiologia da deficiência nutricional de ferro nesse período da vida.

\section{Colaboradores}

A. S. Oliveira participou da concepção do estudo, coleta e análise dos dados, interpretação dos resultados e redação do manuscrito. R. C. R. Silva participou da concepção do estudo, análise dos dados, interpretação dos resultados e redação do manuscrito. R. L. Fiaccone participou da análise dos dados, interpretação dos resultados e redação do manuscrito. E. J. Pinto participou da análise dos dados e revisou o manuscrito. A. M. O. Assis participou da concepção do estudo, interpretação dos resultados e redação do manuscrito.

\section{Agradecimentos}

Ao grupo de nutricionistas que participou da coleta de dados no campo (Andréia Santos de Carvalho, Lívia Karine dos Santos Cruz, Maíra Patriarcha Leal, Soraia Rocha de Brito e Juliana Argolo); à Prefeitura Municipal de Mutuípe e às famílias que participaram do estudo. 


\section{Referências}

1. World Health Organization. Focusing on anaemia: towards an integrated approach for effective anaemia control. Geneva: World Health Organization; 2004.

2. United Nations Children's Fund. The state of the world's children. New York: United Nations Children's Fund; 1998.

3. World Health Organization. Iron deficiency anaemia: assessment, prevention, and control. Geneva: World Health Organization; 2001.

4. Freire WB. Strategies of the Pan American Health Organization/World Health Organization for the control of iron deficiency in Latin America. Nutr Rev 1997; 55:183-8.

5. Oliveira RS, Diniz AS, Benigna MJ, Miranda-Silva SM, Lola MM, Goncalves MC, et al. Magnitude, geographic distribution and trends of anemia in preschoolers, Brazil. Rev Saúde Pública 2002; 36:26-32.

6. Osorio MM, Lira PI, Batista-Filho M, Ashworth A. Prevalence of anemia in children 6-59 months old in the state of Pernambuco, Brazil. Rev Panam Salud Pública 2001; 10:101-7.

7. Assis AMO, Barreto ML, Gomes GSS, Prado MS, Santos NS, Santos LMP, et al. Childhood anemia prevalence and associated factors in Salvador, Bahia, Brazil. Cad Saúde Pública 2004; 20:1633-41.

8. Monteiro CA, Szarfarc SC, Mondini L. Secular trends in childhood in the city of Sao Paulo, Brazil (1984-1996). Rev Saúde Pública 2000; 34(6 Suppl):62-72.

9. Silva LS, Giugliani ER, Aerts DR. Prevalence and risk factors for anemia among children in Brazil. Rev Saúde Pública 2001; 35:66-73.

10. Ferreira HA, Assunção ML, Vasconcelos VS, Melo FP, Oliveira CG, Santos TO. Health of marginalized populations: undernutrition, anemia and intestinal parasitic infections among children of a slum of the "Homeless Movement", Maceió, Alagoas. Rev Bras Saúde Mater Infant 2002; 2:177-85.

11. Lacerda E, Cunha AJ. Iron deficiency anemia and nutrition in the second year of life in Rio de Janeiro, Brazil. Rev Panam Salud Pública 2001; 9: 294-301.

12. Hadler MC, Juliano Y, Sigulem DM. Anemia in infancy: etiology and prevalence. J Pediatr (Rio J.) 2002; 78:321-6.

13. Neves MBP, Silva EMK, Morais MB. Prevalência e fatores associados à deficiência de ferro em lactentes atendidos em um centro de saúde-escola em Belém, Pará, Brasil. Cad Saúde Pública 2005; 21:1911-8.

14. Assis AM, Gaudenzi EN, Gomes G, Ribeiro RC, Szarfarc SC, Souza SB. Hemoglobin concentration, breastfeeding and complementary feeding in the first year of life. Rev Saúde Pública 2004; 38:54351.

15. Lima ACVMS, Lira PIC, Romani SAM, Eickman SH, Piscoya MD, Lima MC. Fatores determinantes dos níveis de hemoglobina em crianças aos 12 meses de vida na Zona da Mata Meridional de Pernambuco. Rev Bras Saúde Matern Infant 2004; 4:35-43.

16. Dallman PR. Iron deficiency and the immune response. Am J Clin Nutr 1987; 46:329-34.
17. Walter T, Andraca I, Chadud P, Perales CG. Iron deficiency anemia: adverse effects on infant psychomotor development. Pediatrics 1989; 84:7-17.

18. Pollitt E. Early iron deficiency anemia and later mental retardation. Am J Clin Nutr 1999; 69:4-5.

19. Lozzof BJ, Jimenez E, Hagen J, Mollen E, Wolf AW. Poorer behavioral and developmental outcome more than 10 years after treatment for iron deficiency in infancy. Pediatrics 2000; 105:51.

20. Tricta Junior D. Anemia fisiológica do lactente. J Pediatr (Rio J.) 1986; 60:105-10.

21. Pastel RA, Howanitz PJ, Oski FA. Iron sufficiency with prolonged exclusive breast-feeding in Peruvian infants. Clin Pediatr (Phila) 1981; 20:625-6.

22. Stekel A. Iron nutrition in infancy and childhood. New York: Nestlé; 1984.

23. Oliveira LPM, Assis AMO, Pinheiro SMC, Prado MS, Barreto ML. Alimentação complementar nos dois primeiros anos de vida. Rev Nutr 2005; 18:459-69.

24. Soares NT, Guimarães ARP, Sampaio HAC, Almeida PC, Coelho RR. Padrão alimentar de lactentes residentes em áreas periféricas de Fortaleza. Rev Nutr 2000; 13:167-76.

25. Szarfarc SC, Souza SB, Furumoto RAV, Brunken GS Assis AMO, Gaudenzi EN, et al. Concentração de hemoglobina em crianças do nascimento até um ano de vida. Cad Saúde Pública 2004; 20:266-74.

26. Oliveira MAA, Osório MM, Raposo MCF. Concentração de hemoglobina e anemia em crianças no Estado de Pernambuco, Brasil: fatores sócio-econômicos e de consumo alimentar associados. Cad Saúde Pública 2006; 22:2169-78.

27. Levy-Costa RB, Monteiro CA. Consumo de leite de vaca e anemia na infância no município de São Paulo. Rev Saúde Pública 2004; 38:797-803.

28. Oliveira MA, Osório MM, Raposo MC. Socioeconomic and dietary risk factors for anemia in children aged 6 to 59 months. J Pediatr (Rio J.) 2007; 83:39-46.

29. Assis AM, Monteiro MC, Santana MLP, Santos NS, Pinheiro SMC, Gomes GSS. Diagnóstico de saúde e nutrição da população de Mutuípe-BA. Salvador: EdUFBA; 2002.

30. Superintendência de Estudos Econômicos. Índice de desenvolvimento econômico e social dos municípios baianos. Salvador: Superintendência de Estudos Econômicos; 2002.

31. Centers for Disease Control and Prevention. Recommendations to prevent and control iron deficiency in the United States. Atlanta: Centers for Disease Control and Prevention; 1998. (Report, 47[RR-3])

32. Organización Panamericana de la Salud/Organización Mundial de la Salud. Indicadores para evaluar las prácticas de lactancia materna. Washington DC: Centro de Estudio y Documentación, Organización Mundial de la Salud; 1991.

33. Organización Mundial de la Salud. Medición del cambio del estado nutricional: directrices para evaluar el efecto nutricional de programas de alimentación suplementaria destinados a grupos vulnerables. Genebra: Organización Mundial de la Salud; 1983. 
34. Lohman TG, Roche AF, Martorell R. Anthropometric standardization reference manual. Champaign: Human Kinetics; 1988.

35. Issler RM, Giugliani ER. Identification of the groups most vulnerable to infant malnutrition through the measuring of poverty level. J Pediatr (Rio J.) 1997; 73:101-5.

36. Costa SC. Modelos lineares generalizados mistos para dados longitudinais [Tese de Doutorado]. Piracicaba: Universidade de São Paulo; 2003.

37. Hosmer DW, Lemeshow S. Applied logistic regression. New York: John Wiley \& Sons; 1989.

38. Ministério da Saúde/Organização Pan-Americana da Saúde. Guia alimentar para crianças menores de 2 anos. Brasília: Ministério da Saúde; 2005.

39. Organización Mundial de la Salud. Necesidades de vitamina A, hierro, folato y vitamina B12. Roma: Organización de las Naciones Unidas para la Agricultura y la Alimentación/Organización Mundial de la Salud; 1991.

40. Dewey KG, Cohen RJ, Rivera LL, Brown KH. Effects of age of introduction of complementary foods on iron status of breast-fed infants in Honduras. Am J Clin Nutr 1998; 67:878-84.

41. Duarte L, Fujimori E, Minagawa A, Schoeps F, Montero R. Aleitamento materno e níveis de hemoglobina em crianças menores de 2 anos em município do estado de São Paulo, Brasil. Rev Nutr 2007; 20:149-57.

42. World Health Organization. Complementary feeding of young children in developing countries: a review of current scientific knowledge. Geneva: World Health Organization; 1998.
43. Dallman PR, Siimes MA, Stekel A. Iron deficiency in infancy and childhood. Am J Clin Nutr 1980; 33:86-118

44. Franco E, Hertrampf E, Rodriguez E, Illanes JC, Palacios L, Llaguno S, et al. Iron nutrition in Mapuche infants fed with human milk (2d phase). Rev Chil Pediatr 1990; 61:248-52.

45. Szarfarc SC, Berg G, Santos AL, de Souza SB, Monteiro CA. Prevention of anemia in the first year of life in health centers of Santo Andre, Sao Paulo. J Pediatr (Rio J.) 1996; 72:329-34.

46. Carvalho MR, Tamez RN. Amamentação: bases científicas para a prática profissional. Rio de Janeiro: Editora Guanabara-Koogan; 2002.

47. Minagawa AT, Oliveira IM, Fujimori E, Laurenti D, Montero RM. Profile of breastfeeding in children under 2 years old in the city of Itupeva, SP, Brazil. Arch Latinoam Nutr 2005; 55:132-9.

48. Vannuchi M, Thomson Z, Escuder M, Tacla M, Vezozzo K, Castro L, et al. Perfil do aleitamento materno em menores de um ano no município de Londrina, Paraná. Rev Bras Saúde Mater Infant 2005; 5:155-62.

49. Oliveira LPM, Assis AMO, Gomes GSS, Prado MS, Barreto ML. Duração do aleitamento materno, regime alimentar e fatores associados segundo condições de vida em Salvador, Bahia, Brasil. Cad Saúde Pública 2005; 21:1519-30.

50. Torres MA, Braga JA, Taddei JA, Nobrega FJ. Anemia in low-income exclusively breastfed infants. J Pediatr (Rio J.) 2006; 82:284-7.

Recebido em 11/Fev/2009

Versão final reapresentada em 25/Nov/2009

Aprovado em 01/Dez/2009 